\title{
Effect of a non-linearity on delays and velocities in a CRLH-MTM transmission line model
}

\author{
Salman Raju Talluri ${ }^{1}$, Sunil Vidhya Bhooshan ${ }^{2}$ \\ Asst. Prof, Dept. of Electronics and Communication Engineering, JUIT, Solan, India ${ }^{1}$ \\ Professor, Dept. of Electronics and Communication Engineering, JUIT, Solan, India ${ }^{2}$
}

\begin{abstract}
The theme of this paper is to analyze the effect of a non-linearity (second order constitutive relationship between voltage and current in resistor, inductor and capacitor)) on phase delay and group delay for a unit cell of a conventional transmission line model and then by analyzing the phase and group velocities in a Composite Right Hand Left Hand Meta-material (CRLH-MTM) structure which is being used very extensively in the Transmission line (TL) theory for negative refractive index material realization. The complex propagation constant is determined for the nonlinear transmission line model and it is observed that the non-linearity in the unit cell is producing the negative resistance which can be used in different electronic circuits, if the same non-linear devices are possible to be modelled from existing devices. Along with this, it is also observed that in order to remove the guard band that exists in the CRLH-MTM for supporting forward and backward waves, there has to be some non-linearity in the transmission line model which is a new finding after the study.
\end{abstract}

Keywords: Transmission line model, dispersion, complex propagation constant, phase velocity and group velocity.

\section{INTRODUCTION}

Realization of Composite right hand left hand meta-materials (CRLH-MTMs) using microstrip is a very active research area in electromagnetics and many devices have been developed so far [1-5]. The main reason of research for these novel circuits is to come up with negative refractive index material or to come up with the conclusion that the phase velocity and group velocity are in opposite direction for a forward travelling wave which is a different phenomenon. But the main problem with these circuits when they realized in the microstrip is that, the gain appears to be very small [6] when the transition is happening from same sign of phase velocity and group velocity to the opposite sign. In this paper it is shown that with the non linearity in the unit cell, it is possible to avoid this difficulty there by opening an opportunity for the nonlinear transmission lines in a better perspective.

Almost all devices exhibit non-linearity depending on conditions of operations. But Non-linear circuits are very common in electronics field like clippers, clampers and harmonic generators. In many cases, if there are any non-linear devices present in any circuit, they are linearized within its operating region designated as bias point or quiescent point using the Taylor's series expansion [7]. In some applications the circuits are supposed to be analysed without any linearization to see the actual effects of non-linearity in the entire system. There are so many different reasons for the non-linearity for any given component. Capacitor with fringing field can be modelled as a non-linear capacitor, inductor on an iron core is a non-linear device and resistor in an environment with changing temperature is considered as non-linear resistance. Sometimes these non-linearities may be very complicated functions of controlling parameters. Even though there are infinitely many possible non-linearities exist for a device, it is appropriate to study the effect of a specific non-linearity for observing the systems behaviour to some extent.

This paper starts with the analysis of a non-linear resistor [8] and its behaviour as a function of frequency. The frequency response [9] is obtained by exciting the non-linear resistor with a Gaussian current pulse by observing the voltage and there by using the Fourier Transform to get both the magnitude response and phase response.

Secondly, an iron core inductor which has a nonlinear relationship between the magnetic flux and current has been analysed. Using the principle of duality [10], similar type of conclusions can be drawn out for the nonlinear capacitor.

Finally, the standard transmission line models [11] are analysed with these non-linear devices for two models, one which support the forward wave supporting structure and the other which supports both forward and backward waves. The effect of this non-linearity on phase response of band pass filter transfer function (which is equivalent to CRLH-MTM in wave theory) has been analysed as a practical case and it is observed that nonlinearity is giving negative group delay.

The next section gives the problem formulation. Section III presents the mathematical analysis, discussion and interpretation of simulation results while Section IV 
gives the conclusions based on the findings from the fundamental analysis.

\section{Problem statement}

The aim of this paper is to analyse the effects of a non-linearity on the phase delay and group delay for forward wave supporting structure and forward-backward supporting structure. Linear circuit analysis is very easy compared with the non-linear circuit analysis. A linear circuit or system's behaviour can be expressed completely in terms of unit impulse response, if it is assumed to be time invariant. This is not the case with non-linear systems. In analysing the non-linear circuits or systems, there are problems like frequency conversion, harmonics generations and it is not possible to come up the output calculation for a given circuit from its impulse response as it is not an LTI system. As far as finding the frequency response for a non-linear device is concerned, depending on the spectrum of input signal, it is possible to find the ratio of output to input, if both the power spectral densities are confined within some reasonable limits like it is defined in half power bandwidth for low pass or high pass filters.

This paper is presenting the analysis for a nonlinearity which is a square relationship between the variables. The relation between voltage and current in a linear resistor is given as $v(t)=R i(t)$. But almost all practical resistors exhibit the non-linearity beyond its ratings. It is a very reasonable assumption to consider this device as linear in its operating region. But to study the behaviour of the non-linearity for this resistance, voltage and current relationship is considered as $v(t)=R_{1} i(t)+$ $R_{2} i(t)^{2}$. This equation is in time domain and using the transform theory, it is possible to convert this relationship into frequency domain to observe its properties over wide range of frequencies.

For an inductor on iron core, the relationship between magnetic flux and current passing through the loop are related as $i(t)=\frac{N \phi(t)}{L}+A \phi(t)^{3}$ where $N$ is the number of turns of the coil, $A$ is the cross sectional area of the core and $L$ is the self inductance. For a linear inductor the coefficient $A=0$. Using the Faraday's law of induction along with Lenz's law, the induced voltage can be found out as $v(t)=-N \frac{d \phi(t)}{d t}$. For a linear inductor the relation between induced voltage and current are related as $v(t)=L \frac{d i}{d t}$. For a non-linear device, it is little difficult to come up with a simple equation of $\phi(t)$ in terms of $i(t)$ not involving radical signs. If the values of $A, N$ and $L$ are known, then with the help of curve fitting it is easy to approxmate the same function and $\phi(t)$ can be expressed as some function of $i(t)$. This is illustrated with the following numbers.

Fig.1 represents this non-linear relation between flux and current with $N=5 ; L=1 H ; A=0.1 m^{2}$ when Copyright to IJARCCE 
Resistor: The voltage for the above considered non-linear resistor is obtained as $v(t)=R_{1} i(t)+R_{2} i(t)^{2}=$ $R_{1} \frac{1}{\sigma \sqrt{2 \pi}} e^{-\frac{1}{2}\left(\frac{t-\varpi}{\sigma}\right)^{2}}+R_{2} \frac{1}{\sigma^{2} 2 \pi} e^{-\left(\frac{t-\varpi}{\sigma}\right)^{2}}$. The Fourier transform for this can be obtained as $V(j \omega)=$ $R_{1} e^{-j \omega \mu} \cdot e^{\frac{-\omega^{2} \sigma^{2}}{2}}+R_{2}^{\prime} e^{-j \omega \mu} \cdot e^{\frac{-\omega^{2} \sigma^{2}}{4}}$. The impedance can be found as the ratio of voltage to current in frequency domain as $Z(j \omega)=\frac{V(j \omega)}{I(j \omega)}=\frac{R_{1} e^{-j \omega \mu} \cdot e^{\frac{-\omega^{2} \sigma^{2}}{2}}+R_{2}^{\prime} e^{-j \omega \mu} \cdot e^{\frac{-\omega^{2} \sigma^{2}}{4}}}{e^{-j \omega \mu} \cdot e^{\frac{-\omega^{2} \sigma^{2}}{2}}}$.

Fig. 2 represents the impedance with different values of $R_{2}^{\prime}$ by keeping $R_{1}$ as a constant.
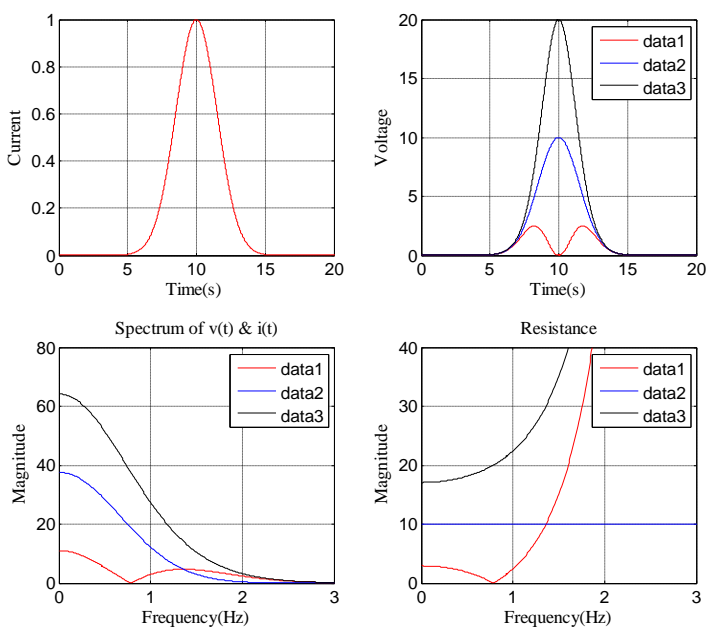

Fig.2 Variation of resistance as a function of frequency. Data $1: R_{2}^{\prime}=$ $-10 \Omega$, Data $2: R_{2}^{\prime}=0 \Omega$ and Data $3: R_{2}^{\prime}=10 \Omega$ with $R_{1}=10 \Omega$

Inductor: The same procedure can be used to find the impedance for the non-linear inductor and those results are communicated by the same authors in detail in [12]. Same arguments can be used for non-linear capacitor as well.

\section{Combinations of inductor and capacitor:}

After studying the effect of non-linearity for each device, the combinations of capacitors and inductors are used in series and shunt configurations. The study is done by considering individually one element as non-linear and then by considering both elements as non-linear.

Non-linear inductor and capacitor in a series circuit gives a higher order non-linear differential equation and this problem is converted into first order differential equations and then solved using the fourth order RungeKutta method to find the currents and voltages in time domain. These signals are converted into the frequency domain by taking the FFT to get the spectrum. Three different combinations are considered. In the first case, only inductor is non-linear. In the second case, only capacitor is taken as non-linear. In the third case, both capacitor and inductor are taken as non-linear.

Fig. 3 represents the variations in the impedance when the inductor has been changed alone. This is observed for three different values and there is a deviation in the phase response of series inductor and capacitor configuration there by introducing an imaginary resistance coming from the non-linearity.
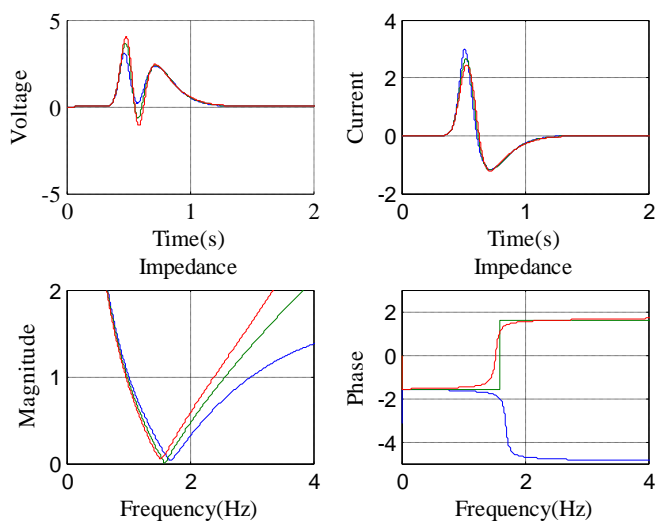

Fig.3 Blue: $L_{2}=-0.02 H$, Green: $L_{2}=0 \mathrm{H}$ Red: $L_{2}=0.02 \mathrm{H}$ with $L_{1}=0.1 H, C_{1}=0.1 F$ and $C_{2}=0 F$

Fig.4 represents the impedance for variations in capacitor.
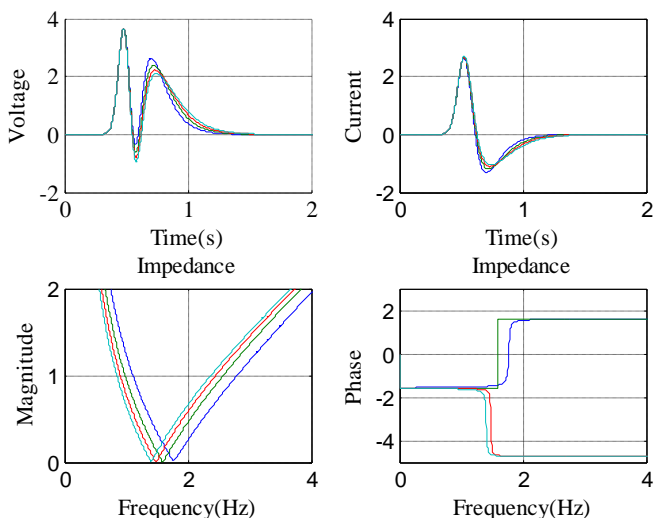

Fig.4 Blue: $C_{2}=-0.01 F$, Green: $C_{2}=0 F$ Red: $C_{2}=0.01 F$ Cyan: $C_{2}=0.02 F$ with $L_{1}=0.1 \mathrm{H}, C_{1}=0.1 \mathrm{~F}$ and $L_{2}=0 \mathrm{H}$.

Fig.5 represents the analysis results for both capacitor and inductor non-linearities.
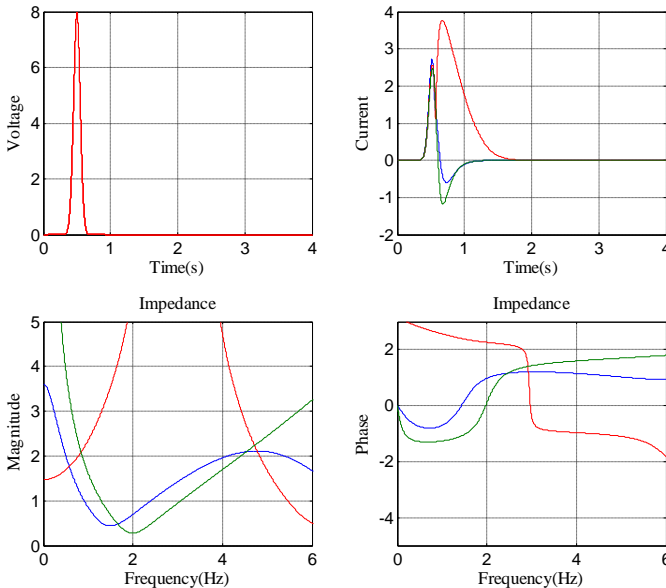

Fig.5 Blue: $L_{2}=-0.01 H, C_{2}=-0.02 F$, Red: $L_{2}=0.0 H, C_{2}=$ $0 F$, Green: $L_{2}=0.01 \mathrm{H}, C_{2}=0.02 \mathrm{~F}$ all with $L_{1}=0.1 \mathrm{H}, C_{1}=0.1 \mathrm{~F}$ and $C_{2}=0 F$ 

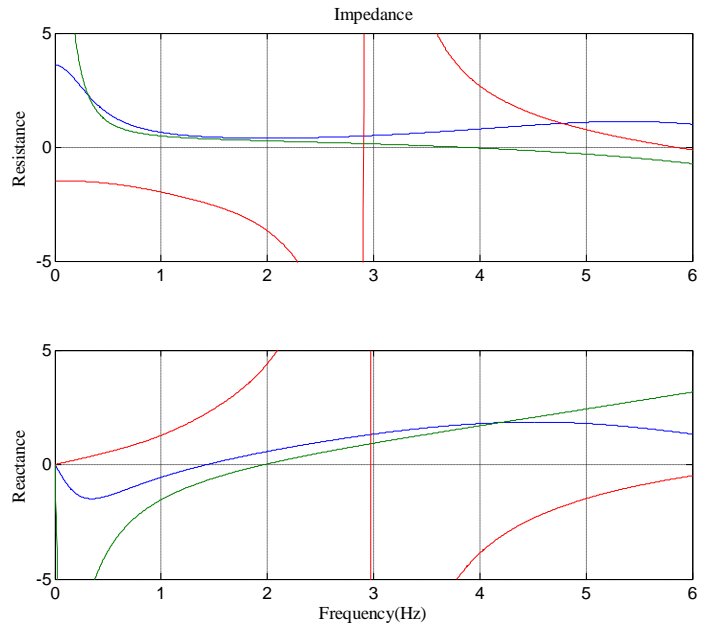

Fig.6 Same values with Fig.5

Fig.6 is another way of representing the impedance that is obtained from Fig.5 to actually see that the negative resistance has been produced due to nonlinearity. This is compared with the series impedance of $R L C$ circuit with positive resistance and negative resistance to validate the arguments. Fig.7 is the phase characteristic of a series inductor and capacitor along with resistance. Usually resistance is positive and the blue line represents the actual behaviour with positive resistance. The red trace represents the phase characteristic with negative resistance.

In Phasor notation for circuit analysis, first and fourth quadrants are used to represent the linear elements but with the presence of non-linear elements in a given circuit, it is necessary to use the second and third quadrant as well. From the real part of impedance for the series combination of capacitor and inductor, an equivalent circuit can be obtained at different frequencies which exhibiting the negative resistance in its frequency behaviour.

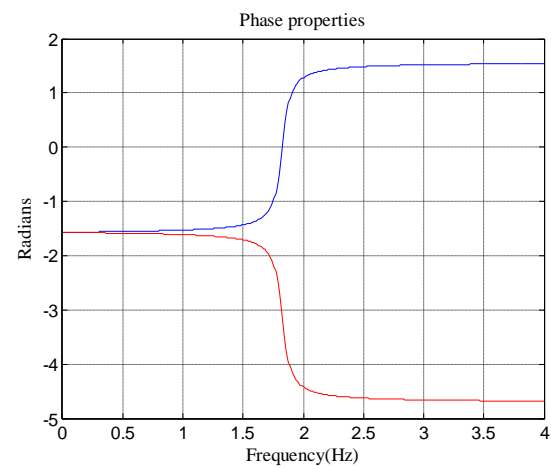

Fig.7 Series RLC circuit response for positive and negative resistances.

After this, in order to observe the effect of nonlinearity on phase response of a low pass transmission line model (which is a forward supporting wave structure) is considered. This is analysed in the circuit theory (to find the gain) and in wave theory (to find the complex propagation constant).Fig. 8 is for the lowpass structure and Fig. 9 is for bandpass structure in circuit theory to find the gain of the entire structure.
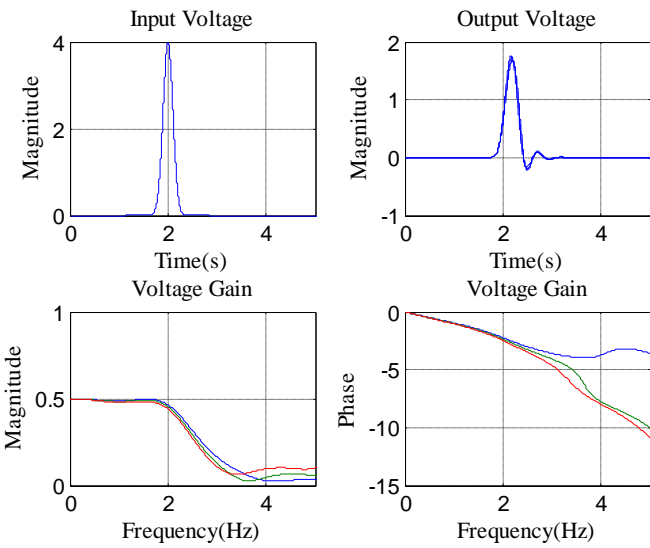

Fig.8 Lowpass structure
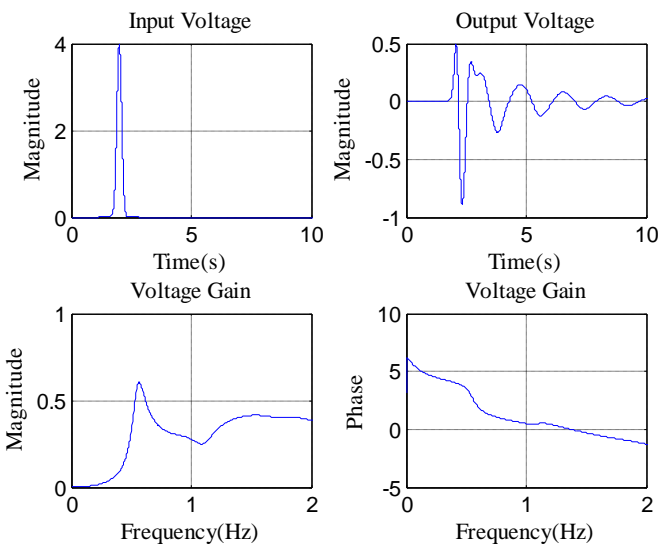

Fig.9 Bandpass structure.

Fig.10 and Fig. 11 are the characteristics in wave theory to support that there has to be some non-linearity in the equivalent model to have a smooth transition from backward to forward wave supporting structure.
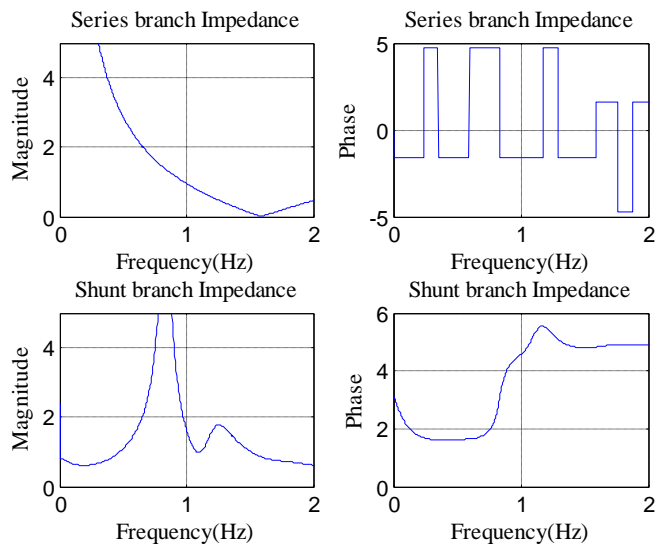

Fig.10 Impedances for series branch and shunt branches. 

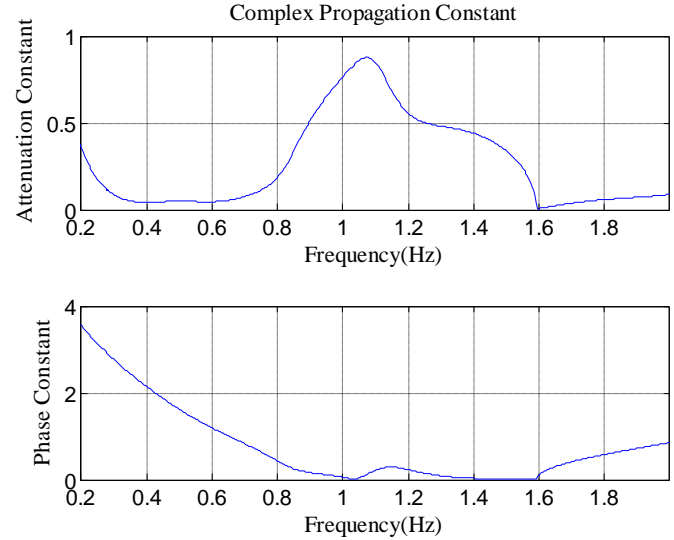

Fig.11 Attenuation constant from wave theory.

\section{CONCLUSION}

After the analysis is carried out, it is observed that even a small non-linearity in the unit cell can cause problems in stability. The problem with the non-linear devices is that they produce harmonics and other effects which can lead to instability in the given circuit. From the simulations, it is observed that the non-linearity in the series combination of inductor and capacitor produces some negative resistance. For the lowpass and bandpass filter characteristics, there is maxima/minima in the phase characteristics for the gain of the unit cell there by indicating the opposite phase delay and group delay. These delays can be translated in to phase velocity and group velocities in the wave theory there by giving a possibility of negative refractive index materials with the non-linear elements. In case if the non-linearity can be controlled in a precise manner, these non-linearities can be used in many electronic circuits.

\section{REFERENCES}

11 A. Lai, C. Caloz, and T. Itoh, "Composite right/left-handed transmission line metamaterials," IEEE Microw. Mag., vol. 5, no. 3, pp. 34-50, Sep. 2004

[2] C. Caloz, H. Okabe, T. Iwai, and T. Itoh, "Transmission line approach of left-handed (LH) materials," in Proc. USNC/URSI National radio Science Meeting, San Antonio, TX, June 2002, vol. 1, p. 39.

[3] C. Caloz and T. Itoh, "Novel microwave devices and structures based on the transmission line approach of meta-materials," in Proc. IEEE-MTT Int. Symp., Jun. 2003, vol. 1, pp. 195-198.

[4] G.V. Eleftheriades, O. Siddiqui, and A.K. Iyer, "Transmission

line models for negative refractive index media and associated implementations without excess resonators," IEEE Microwave Wireless Compon. Lett., vol. 13, pp. 51-53, Feb. 2003.

[5] G.V. Eleftheriades, A.K. Iyer, and P.C. Kremer, "Planar negative refractive index media using periodically L-C loaded transmission lines," IEEE Trans. Microwave Theory Tech., vol. 50, pp. 27022712, Dec. 2002..

[6] G. V. Eleftheriades and K. G. Balmain, Negative-Refraction Metamaterials:Fundamental Principles and Applications. NewYork,NY, USA: Wiley, 2005.

[7] Erwin Kreyszig, Advanced Engineering Mathematics, Wiley India Private Limited, 2006

[8] Halliday, Resnick and Walker, Fundamentals of Physics Electricity and Magnetism, Wiley India Private Limited, 2011

[9] Alan Victor Oppenheim, Alan S. Willsky, with S. Hamid Nawab, Signals and Systems, Prentice-Hall International, 1997.

[10] William Hart Hayt, William Hayt, Jack E.Kimmerly and Steven M.Durbin, Enginnering Circuit Analysis. New York: MCGRAWHILL higher Education, 2012.
[11] S. Ramo, J. R. Whinnery, and T. Van Duzer, Fields and Waves in communication Electronics, 3rd ed., New York, NY: Wiley, 1994

[12] Salman Raju Talluri,Sunil Vidya Bhooshan, "Fundamental study of a non-linear capacitor to use it in non-linear transmission line models",IJARCCE, volume 3,Issue 7,July 2014.

[13] MATLAB version 7.10.0.499 (R2010a).Natick, Massachusetts: The Math Works Inc., 2010. 